\title{
Ferramenta de Checagem do Ponto de Operação de Bombas Utilizando Plataforma Labview
}

\author{
Tool Checking The Pump Operation Point Using Labview Platform
}

\section{Heberth de Carvalho Pereira, Alex Franco Ferreira*}

Como citar esse artigo. Pereira, H.C; Ferreira, A.F. Ferramenta de checagem do ponto de operação de bombas utilizando plataforma Labview. Revista Teccen. 2021 Jan./Jun.; 14 (1): 14-18.

\section{Resumo}

Em uma indústria do ramo papeleiro, o processo de fabricação envolve uma grande quantidade de bombas de fluídos líquidos necessários para a formação adequada do papel, baseado nisso, é fundamentalconhecer a real capacidade de bombeamento verificando o ponto de operação das mesmas. Este trabalho tem a finalidade de demonstrar comofoi desenvolvida a ferramenta para tal verificação. Trata-se de um programa baseado na plataforma LabView com a função de adquirir, condicionar e registrar os dados provenientes do campo fabril, permitindo a medição do rendimento da bomba. O funcionamento da aplicação retornou com resultados que permitiram a constatação da região de operação, a mesma trabalha muito próximo dos $60 \%$ de rendimento, permitiu ainda a visualização de que ainda existe capacidade de ampliar a vazão, o que proporciona novas oportunidades para o setor de desenvolvimento de produto com papéis que consomem maior quantidade de insumos.

Palavras-chave: : LabView; ponto de operação; bombas.

\begin{abstract}
In a paper industry, the manufacturing process involves a large number of liquid fluid pumps necessary for the proper formation of paper, based on this, it is essential to know the real pumping capacity by checking their point of operation. This work aims to demonstrate how the tool for such verification was developed. It is a program based on the LabView platform with the function of acquiring, condition in gand recording data from the manufacturing field, allowing the measurement of pump performance. The operation of the application returned with results that allowed the verification of the region of operation, it works very close to $60 \%$ of yield, it also allowed the visualization that there is still the capacity to expand the flow, which provides new opportunities for the sector of product development with papers that consume more inputs.

Keywords: LabView; Point of Operation; Pumps.
\end{abstract}

\section{Introdução}

Em uma indústria papeleira, parte do processo de fabricação baseia-se na aplicação de produtos, muitas vezes preparado na própria planta, na composição do papel. Estes elementos são indispensáveis à fabricação pois o alcance de algumas características referentes a qualidadetais como tração, alvura, permeabilidade e até andamento de máquina, estão associadas aos mesmos (Castro, 2009).

Sendo assim, um controle preciso e estável dos produtos e subprodutos associados ao papel é necessário para evitar a geração de refugo nas máquinas. Este trabalho tem por objetivo realizar uma inspeção nos elementos de controle, especificamente os que se utilizam de bombas, e verificar se a região de operação proposta pela receita da máquina é tolerável pelo equipamento, de forma que sua performance e estabilidade não sejam afetadas.

A tendência do negócio é diversificar cada vez mais a produção (Paiva, Jr, \& Fensterseifer, 2009) para a manutenção, sobrevivência e conquista de novos clientes, para isso, é necessário conhecer os limites do nosso processo.

A ferramenta a ser utilizada para essa checagem chama-se LabVIEW (Laboratory Virtual InstrumentEngineering Workbench), que segundo Corporation, National Instruments(2000), é uma 
plataforma computacional que desenvolve programas para aquisição de dados do campo fabril, realiza o tratamento dessa informação, registra possibilitando a análise e geração de relatórios.

O conhecimento desses limites e de qual é a região de operação dasbombas auxilia a tomada de decisão gerencial e comercial. A checagem do ponto de operação atual de uma bomba centrífuga, que é a mais utilizada, permite confrontar com o ponto ou região em que ela foi projetada, sendo possível avaliar sua eficiência e validar se está prestando um bom atendimento à máquina. Sendo assim o presente trabalho contribui para a criação eimplementação de um sistema capaz de verificar os indicadores fundamentais da bomba, necessários para medir sua eficiência e somente assim, confrontar com o que foi proposto pelo fabricante ou projeto.

\section{Material e Métodos}

Foi desenvolvido para atender a esta proposta um programa utilizando o software LabVIEW, que tivesse a capacidade de monitorar o ponto de operação da bomba, seja ela qual for utilizando-se da curva fornecida pelo fabricante como referência, como mostra a Figura 1. A partir desse monitoramento, foi gerado um histórico de vazão para análise, que permitiu uma avaliação do equipamento.

A bomba em questão é do modelo INI-O 100-400 do fabricante IMBIL, trabalha numa rotação máxima de 1160RPM, o rotor tem um diâmetro de $390 \mathrm{~mm}$. $\mathrm{O}$ acionamento é realizado por um motor de $60 \mathrm{CV}, 4$ polos, 1775RPM, modelo 225SM0790 alimentado por um inversor de frequência que trabalha próximo de $42 \mathrm{~Hz}$. Segundo IMBIL - Soluções em Bombeamento (2020), o rotor tipo O é recomendado para bombear fluidos compostos de líquidos e ar, como resíduos sem bagaço de usinas de cana de açúcar e massa de papel.

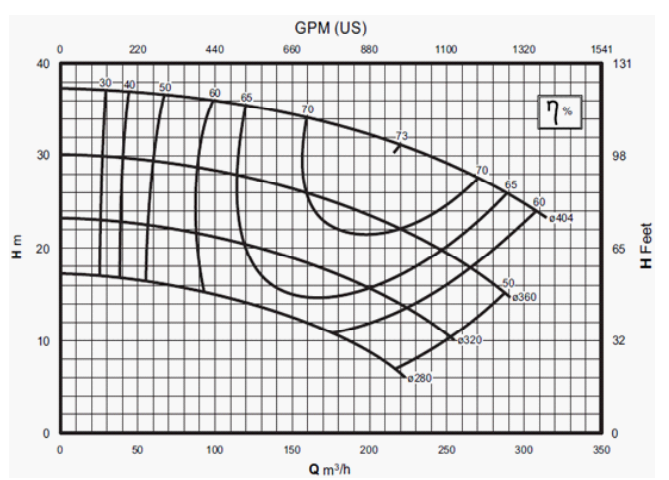

Figura 1. Curva de Rendimento da bomba INI-O $100-400$.

Fonte: IMBIL - Soluções em Bombeamento, 2020.

\section{O LabVIEW}

Software utilizado para criação de aplicações cuja programação não se baseia em linhas de texto e sim num ambiente gráfico onde as funções são apresentadas como ícones, tornando-se uma linguagem favorável ao aprendizado e à visualização do fluxo de dados circulante na lógica programada. Para Sumathi \& Surekha (2007), o LabVIEW permitiu a engenheiros criar recursos que antes só era possível a profundos conhecedores de linguagens específicas e aos programadores da tecnologia de informação.

A linguagem de programação utilizada é a " $G$ ", que conforme Vilela, Medeiros, \& Piazza (2014), é eficiente na elaboração de sistemas de aquisição de dados, controle e instrumentação, permitindo a criação de interface amigável ao usuário, além de poder ser reaproveitada em outros softwares. Com este sistema, é possível inserir dados físicos como pressão, vazão, distância, frequência, amplitudes de som e luz, tensão, corrente, potência entre outros por meio de placas $\mathrm{AD}$ (analógico/digital) ou DA (digital/analógico), chamadas de DAQ (data Aquisition), protocos de comunicação OPC (OLE for Process Control), instrumentos e transdutores com interfaces GPIB (General Purpose Interface Bus) e Serial (RS232, RS485) entre demais módulos de simulação, cálculo e automação (Sumathi $\&$ Surekha, 2007).

\section{O Protocolo OPC}

Segundo Carvalho, Silva, \& Nascimento (2020), os processos industriais se tornam cada vez mais complexos em busca de maior qualidade do produto. Maior complexidade gera maior quantidade de dados para gerenciar. A tecnologia OPC surgiu da necessidade de integração dos dados processuais nos três níveis de controle (estratégico, tático e operacional), os dispositivos de campo, sistemas de controle e softwares para gerenciamento dos negócios.

As especificações deste protocolo foram fornecidas pela $O P C$ Foundation, para que integrasse aos anceios gerais da indústria e não para que atendesse a um grupo de fabricantes específicos. O protocolo foi criado a partir da tecnologia Microsoft OLE/ COM (protocolos que deram os primeiros passos para a integração de dados em diferentes aplicações). Basicamente, a tecnologia OPC conecta diferentes aplicações de controle de processo em um sistema operacional, IHM (interface homem-máquina) e Sistema supervisório (SCADA - Supervisory Control and Data Aquisition).

De acordo com Carvalho, Silva, \& Nascimento (2020), existem dois componentes OPC, o cliente e o servidor.O cliente é a aplicação que solicita dados, como um sistema supervisório. O servidor é quem 
disponibiliza os dados coletados no campo.Neste trabalho, a aquisição de dados da aplicação criada foi realizada através do servidor OPC.

\section{O programa}

O programa é separado em duas partes: $\mathrm{O}$ Painel Frontal, pode ser visto na Figura 2, é a interface com o usuário, é a camada superior da aplicação onde visualiza-se as indicações das variáveis monitoradas e itens de controle como um botão, um setpoint, gráficos de tendência entre outros.

É a Interface elaborada para interação com o usuário, dispondo dos elementos gráficos que permitirão o acompanhamento do processo em tempo real.

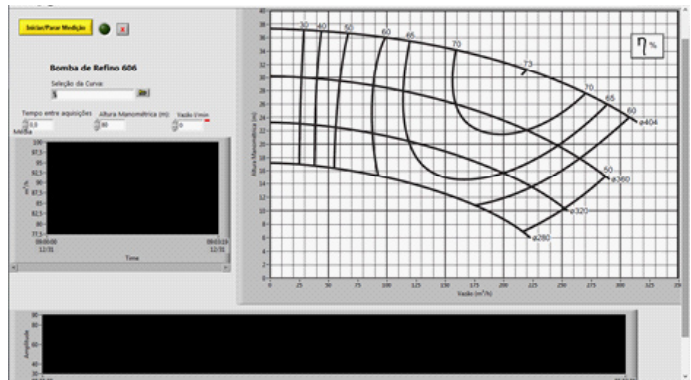

Figura 2. Painel Frontal.

Fonte: Autores, 2020

O Painel de Controle, demonstrado na Figura 3, é a camada inferior da aplicação, é nela que a lógica de funcionamento é desenvolvida, é onde a aquisição de dados foi configurada e tratada para exibição ao usuário.

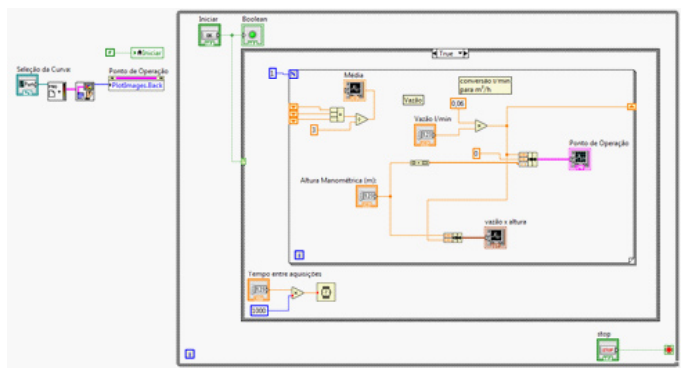

Figura 3. Painel de Controle .

Fonte: Autores, 2020.

Nesta proposta de programa, o tempo de aquisição foi configurado para 1 segundo. Isso significa que a cada segundo, o valor de vazão da bomba foi adquirido e armazenado, formando uma tendência. Foi por meio dessa série de dados que se tornou possível a avaliaçãoda região de operação da bomba.

Na empresa, adota-se como padrão em boa parte do processo, a unidade de medida de vazão em Litros por minuto $(\mathrm{L} / \mathrm{min})$; como a curva do fabricante utilizase de vazão em metros cúbicos por hora $\left(\mathrm{m}^{3} / \mathrm{h}\right)$, foi inserido um bloco para fazer a conversão.Na Figura
4 encontra-se uma decomposição das funções básicas para compreensão do funcionamento da aplicação.

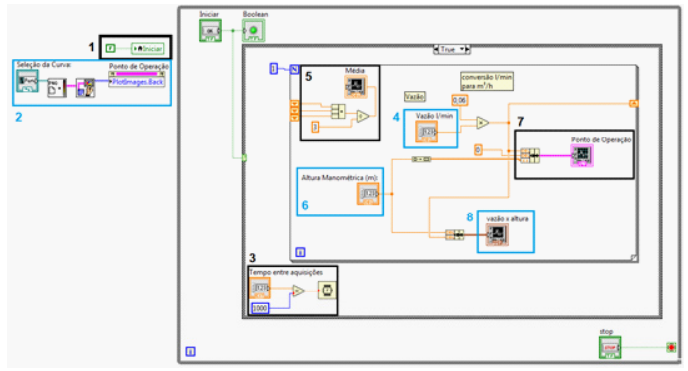

Figura 4. Decomposição da lógica de funcionamento da aplicação.

Fonte: Autores, 2020

1) Garante que sempre que o programa for executado, é necessário que o usuário pressione o botão iniciar para que o software prossiga com a próxima etapa.

2) Função que seleciona a curva para que seja plotada como plano de fundo do gráfico.

3) Seleção do tempo de aquisição de dados, com resolução em milissegundos.

4) Valor de Vazão em Litros por minuto, proveniente de um servidor OPC conectado a um dos Controladores Lógicos Programáveis (CLP) da máquina.

5) Cálculo da média móvel, função que considera sempre os últimos três valores adquiridos. Basicamente, é uma pilha com 3 dados, o primeiro dado a entrar é o primeiro a sair, ou seja, no $4^{\circ}$ segundo de execução, o valor obtido no primeiro sairá da pilha para que o quarto valor seja inserido.

6) Este dado é inserido manualmente, através do painel frontal, visto que a altura manométrica é constante neste caso, medindo $34 \mathrm{~m}$.

7) Após o valor adquirido pelo servidor OPC ser convertido em $\mathrm{m}^{3} / \mathrm{h}$, esta função plota na curva da bomba, fornecendo uma análise instantânea da região em que a bomba está operando, como mostra a Figura 5.

8) Esta função armazena os dados adquiridos, possibilitando a transferência para outros softwares de análise.

Analisando-se a Figura 5, é possível visualizar o que foi explicitado no item 7 do parágrafo anterior, onde o ponto de operação neste instante foi de aproximadamente $90 \mathrm{~m}^{3} / \mathrm{h}$ em $34 \mathrm{~m}$. Dessa forma, avalia-se que no dia dessa medição, 26 de agosto de 2020 , o rendimento era próximo de $60 \%$.

A Tabela 1 foi construída a partir dos dados adquiridos e armazenados pelo sistema. Nela encontram-se uma parte da população que representaos resultadosobtidos no dia 26 de agosto de 2020 . Na primeira e segunda coluna estão registrados o instante da aquisição, já na terceira, a altura manométrica, que 


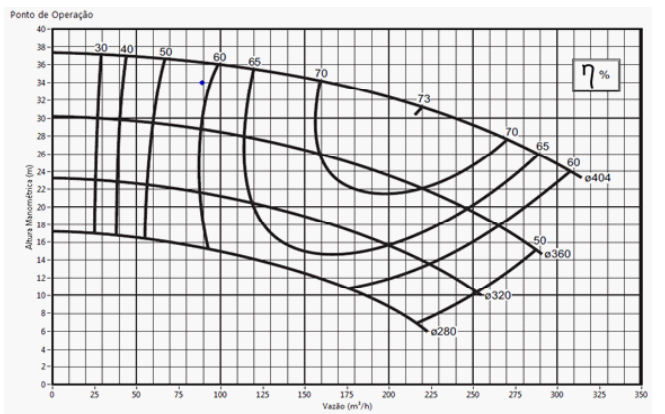

Figura 5. Plotagem do Ponto de Operação da Bomba.

Fonte: Autores, 2020

Tabela 1. Amostragem de 26 de agosto de 2020.

\begin{tabular}{|c|c|c|c|c|}
\hline Data & Horário & $\begin{array}{c}\text { Altura } \\
\text { manométrica } \\
\text { (m) }\end{array}$ & $\begin{array}{l}\text { Vazão } \\
\left(\mathrm{m}^{3} / \mathbf{h}\right)\end{array}$ & $\begin{array}{c}\text { Vazão } \\
\text { (L/min) }\end{array}$ \\
\hline $26 / 08 / 2020$ & $00: 14: 00$ & 34 & 88,9256 & 1482,09 \\
\hline $26 / 08 / 2020$ & 00:14:01 & 34 & 88,8587 & 1480,98 \\
\hline $26 / 08 / 2020$ & 00:14:02 & 34 & 88,8412 & 1480,69 \\
\hline $26 / 08 / 2020$ & 00:14:03 & 34 & 88,8689 & 1481,15 \\
\hline $26 / 08 / 2020$ & $00: 14: 04$ & 34 & 88,9126 & 1481,88 \\
\hline $26 / 08 / 2020$ & $00: 14: 05$ & 34 & 88,9884 & 1483,14 \\
\hline $26 / 08 / 2020$ & 00:14:06 & 34 & 89,0423 & 1484,04 \\
\hline $26 / 08 / 2020$ & $00: 14: 07$ & 34 & 89,1366 & 1485,61 \\
\hline $26 / 08 / 2020$ & 00:14:08 & 34 & 89,1769 & 1486,28 \\
\hline $26 / 08 / 2020$ & 00:14:09 & 34 & 89,1596 & 1485,99 \\
\hline $26 / 08 / 2020$ & $00: 14: 10$ & 34 & 89,3118 & 1488,53 \\
\hline $26 / 08 / 2020$ & $00: 14: 11$ & 34 & 89,3652 & 1489,42 \\
\hline $26 / 08 / 2020$ & $00: 14: 12$ & 34 & 89,4026 & 1490,04 \\
\hline $26 / 08 / 2020$ & $00: 14: 13$ & 34 & 89,4091 & 1490,15 \\
\hline $26 / 08 / 2020$ & $00: 14: 14$ & 34 & 89,3658 & 1489,43 \\
\hline $26 / 08 / 2020$ & $00: 14: 15$ & 34 & 89,3272 & 1488,79 \\
\hline $26 / 08 / 2020$ & $00: 14: 16$ & 34 & 89,3196 & 1488,66 \\
\hline $26 / 08 / 2020$ & $00: 14: 17$ & 34 & 89,3211 & 1488,69 \\
\hline $26 / 08 / 2020$ & $00: 14: 18$ & 34 & 89,3654 & 1489,42 \\
\hline $26 / 08 / 2020$ & 00:14:19 & 34 & 89,4297 & 1490,50 \\
\hline $26 / 08 / 2020$ & $00: 14: 20$ & 34 & 89,4638 & 1491,06 \\
\hline $26 / 08 / 2020$ & $00: 14: 21$ & 34 & 89,5178 & 1491,96 \\
\hline $26 / 08 / 2020$ & $00: 14: 22$ & 34 & 89,5466 & 1492,44 \\
\hline $26 / 08 / 2020$ & $00: 14: 23$ & 34 & 89,5200 & 1492,00 \\
\hline $26 / 08 / 2020$ & $00: 14: 24$ & 34 & 89,4998 & 1491,66 \\
\hline $26 / 08 / 2020$ & $00: 14: 25$ & 34 & 89,5011 & 1491,69 \\
\hline $26 / 08 / 2020$ & $00: 14: 26$ & 34 & 89,4610 & 1491,02 \\
\hline $26 / 08 / 2020$ & $00: 14: 27$ & 34 & 89,4558 & 1490,93 \\
\hline $26 / 08 / 2020$ & $00: 14: 28$ & 34 & 89,4555 & 1490,93 \\
\hline $26 / 08 / 2020$ & $00: 14: 29$ & 34 & 89,4562 & 1490,94 \\
\hline $26 / 08 / 2020$ & $00: 14: 30$ & 34 & 89,4533 & 1490,89 \\
\hline $26 / 08 / 2020$ & $00: 14: 31$ & 34 & 89,4753 & 1491,26 \\
\hline $26 / 08 / 2020$ & $00: 14: 32$ & 34 & 89,4954 & 1491,59 \\
\hline $26 / 08 / 2020$ & $00: 14: 33$ & 34 & 89,5820 & 1493,03 \\
\hline $26 / 08 / 2020$ & $00: 14: 34$ & 34 & 89,7146 & 1495,24 \\
\hline $26 / 08 / 2020$ & $00: 14: 35$ & 34 & 89,7633 & 1496,06 \\
\hline
\end{tabular}

Fonte: Autores, 2020. neste caso é fixa, medindo $34 \mathrm{~m}$. A quarta coluna é composta do valor de vazão $\mathrm{em} \mathrm{m}^{3} / \mathrm{h}$, convertido dos valores de vazão em L/min da quinta coluna, obtidos através do servidor OPC.

A Figura 6 demonstra a plotagem dos dados da Tabela 1 em um gráfico de linhas onde no eixo vertical tem-se a vazão obtida do campo fabril, em L/min (lado esquerdo) e $\mathrm{em} \mathrm{m}^{3} / \mathrm{h}$ (lado direito), ambos em função do tempo em que foram adquiridos.

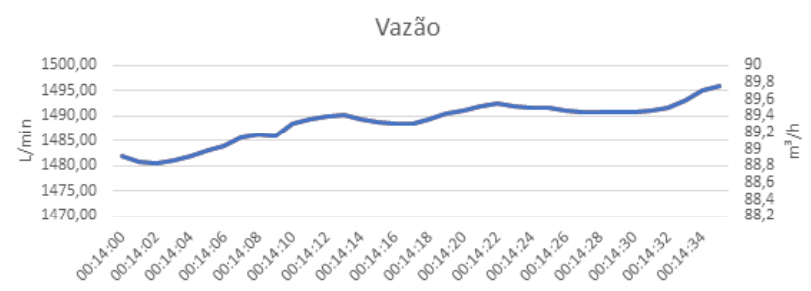

Figura 6. Demonstração Gráfica da Tabela 1

Fonte: Autores, 2020.

A Tabela 2 expõe uma parte da população da média móvel, $5^{\circ}$ item da Figura 4, calculada a partir do monitoramento realizado no dia 09 de setembro de 2020 , com outro tipo de produto em linha de produção. $\mathrm{Na}$ primeira e segunda coluna é registrado e decomposto, em data e hora, o instante do cálculo, a terceira coluna é o valor de vazão em $\mathrm{m}^{3} / \mathrm{h}$, calculado a partir das aquisições via OPC e a quarta coluna, é o valor de vazão convertido em $\mathrm{L} / \mathrm{min}$.

Tabela 2. Média móvel do dia 09 de setembro de 2020.

\begin{tabular}{|c|c|c|c|}
\hline Data & Horário & $\begin{array}{c}\text { Média Vazão } \\
\left(\mathbf{m}^{3} / \mathbf{h}\right)\end{array}$ & $\begin{array}{c}\text { Média Vazão } \\
\text { (L/min) }\end{array}$ \\
\hline $09 / 09 / 2020$ & $09: 11: 25$ & 89,4840 & 1491,40 \\
\hline $09 / 09 / 2020$ & $09: 11: 26$ & 89,4995 & 1491,66 \\
\hline $09 / 09 / 2020$ & $09: 11: 27$ & 89,5276 & 1492,13 \\
\hline $09 / 09 / 2020$ & 09:11:28 & 89,5418 & 1492,36 \\
\hline 09/09/2020 & 09:11:29 & 89,5301 & 1492,17 \\
\hline 09/09/2020 & 09:11:30 & 89,5450 & 1492,42 \\
\hline $09 / 09 / 2020$ & 09:11:31 & 89,5389 & 1492,32 \\
\hline $09 / 09 / 2020$ & $09: 11: 32$ & 89,5364 & 1492,27 \\
\hline $09 / 09 / 2020$ & 09:11:33 & 89,5036 & 1491,73 \\
\hline 09/09/2020 & 09:11:34 & 89,4943 & 1491,57 \\
\hline $09 / 09 / 2020$ & 09:11:35 & 89,4939 & 1491,57 \\
\hline $09 / 09 / 2020$ & $09: 11: 36$ & 89,4996 & 1491,66 \\
\hline $09 / 09 / 2020$ & 09:11:37 & 89,5151 & 1491,92 \\
\hline $09 / 09 / 2020$ & 09:11:38 & 89,5356 & 1492,26 \\
\hline $09 / 09 / 2020$ & 09:11:39 & 89,5487 & 1492,48 \\
\hline $09 / 09 / 2020$ & 09:11:40 & 89,5536 & 1492,56 \\
\hline 09/09/2020 & 09:11:41 & 89,5639 & 1492,73 \\
\hline 09/09/2020 & 09:11:42 & 89,5931 & 1493,22 \\
\hline 09/09/2020 & 09:11:43 & 89,6184 & 1493,64 \\
\hline $09 / 09 / 2020$ & $09: 11: 44$ & 89,6336 & 1493,89 \\
\hline
\end{tabular}


Tabela 2 (cont). Média móvel do dia 09 de setembro de 2020.

\begin{tabular}{|c|c|c|c|}
\hline Data & Horário & $\begin{array}{c}\text { Média Vazão } \\
\left(\mathbf{m}^{\mathbf{3} / \mathbf{h})}\right.\end{array}$ & $\begin{array}{c}\text { Média Vazão } \\
(\mathbf{L} / \mathbf{m i n})\end{array}$ \\
\hline $09 / 09 / 2020$ & $09: 11: 45$ & 89,6356 & 1493,93 \\
\hline $09 / 09 / 2020$ & $09: 11: 46$ & 89,6234 & 1493,72 \\
\hline $09 / 09 / 2020$ & $09: 11: 47$ & 89,6172 & 1493,62 \\
\hline $09 / 09 / 2020$ & $09: 11: 48$ & 89,6192 & 1493,65 \\
\hline $09 / 09 / 2020$ & $09: 11: 49$ & 89,6377 & 1493,96 \\
\hline $09 / 09 / 2020$ & $09: 11: 50$ & 89,7301 & 1495,50 \\
\hline $09 / 09 / 2020$ & $09: 11: 51$ & 89,9329 & 1498,88 \\
\hline $09 / 09 / 2020$ & $09: 11: 52$ & 90,0664 & 1501,11 \\
\hline $09 / 09 / 2020$ & $09: 11: 53$ & 90,1189 & 1501,98 \\
\hline $09 / 09 / 2020$ & $09: 11: 54$ & 90,0488 & 1500,81 \\
\hline $09 / 09 / 2020$ & $09: 11: 55$ & 90,0157 & 1500,26 \\
\hline $09 / 09 / 2020$ & $09: 11: 56$ & 89,9536 & 1499,23 \\
\hline $09 / 09 / 2020$ & $09: 11: 57$ & 89,8737 & 1497,90 \\
\hline $09 / 09 / 2020$ & $09: 11: 58$ & 89,7982 & 1496,64 \\
\hline $09 / 09 / 2020$ & $09: 11: 59$ & 89,7508 & 1495,85 \\
\hline $09 / 09 / 2020$ & $09: 12: 00$ & 89,7100 & 1495,17 \\
\hline $09 / 09 / 2020$ & $09: 12: 01$ & 89,7100 & 1495,17 \\
\hline $09 / 09 / 2020$ & $09: 12: 02$ & 89,6766 & 1494,61 \\
\hline $09 / 09 / 2020$ & $09: 12: 03$ & 89,6187 & 1493,64 \\
\hline $09 / 09 / 2020$ & $09: 12: 04$ & 89,5802 & 1493,00 \\
\hline $09 / 09 / 2020$ & $09: 12: 05$ & 89,5813 & 1493,02 \\
\hline
\end{tabular}

Fonte: Autores, 2020.

A Figura 7 apresenta o gráfico da média móvel com os dados da Tabela 2. O eixo primário (a esquerda) está $\mathrm{em} \mathrm{m}^{3} / \mathrm{h}$ e o secundário (a direita) em L/min.

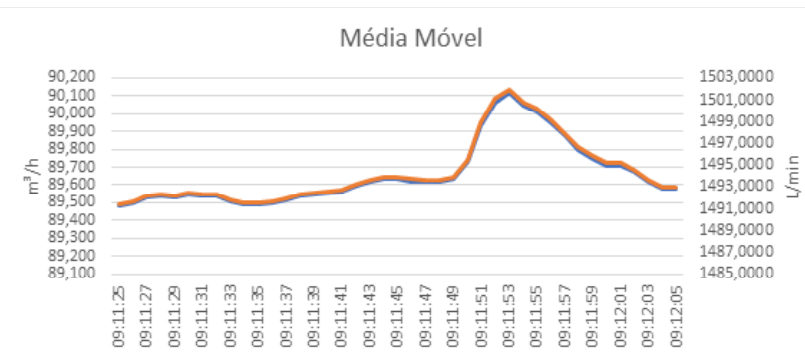

Figura 7. Demonstração Gráfica da média móvel do dia 09 de setembro de 2020.

Fonte: Autores, 2020.

\section{Conclusão}

A aplicação em questão mostrou-se eficiente como ferramenta de avaliação do desempenho de bombas e como registrador de dados. O LabView, associado a um servidor OPC, mostrou-se muito sólido, com requisitos que computadores simples do mercado possam atender, o que proporciona baixo custo de implementação.

O sistema será distribuído em outras máquinas para verificação de suas respectivas bombas. Os dados levantados nestes dois períodos provam que, atualmente, a bomba em questão trabalha numa região bem definida, com rendimento próximo de $60 \%$. A checagem e verificação do ponto de operação foi um sucesso, valida-se a partir de agora, que a bomba trabalha em uma faixa tolerável nas receitas de fabricação e que possui capacidade de vazão maior do que a atual, o que significa que o nicho de mercado de papéis com maior gramatura, o que solicita uma vazão de massa superior ao que foi medido, pode ser explorado pelo nível estratégico da empresa.

Para um maior aproveitamento do sistema, aconselha-se aumentar o período de aquisição, para que não se crie um banco de dados muito extenso, dificultando assim uma análise. Para uma verificação mais precisa e com maior resolução, sugere-seassociação de um banco de dados SQL, possibilitando o armazenamento de um grande volume de informação sem extinguir a capacidade de processamento.

\section{Referências Bibliográficas}

Carvalho, A. S., Silva, R. c., \& Nascimento, D. B. (s.d.). Sistema de Comunicação OPC para uma Coluna de Destilação Piloto. Acesso em 6 de Setembro de 2020, retirado de Associação Educacional Dom Bosco: https://www.aedb.br/seget/arquivos/artigos08/326 Sistema\%20 de $\% 20$ Comunicacao $\% 20$ OPC $\% 20$ para $\% 20$ uma $\% 20$ Coluna $\% 20$ de $\% 20$ Destilacao\%20Piloto.pdf

Castro, H. F. (2009). Processos Químicos Industrias II - Papel e Celulose. Acesso em 8 de Agosto de 2020, retirado de https://sistemas.eel.usp.br/ docentes/arquivos/5840556/434/apostila4papelecelulose.pdf

Corporation, National Instruments. (2000). Manual de treinamento do LabVIEW (Outubro de 2000 ed.).

IMBIL - Soluções em Bombeamento. (20 de Setembro de 2020).

NDBOMBAS. Fonte: https://www.ndbombas.com.br/downloads/Catalogo_ INI-K-O.pdf

Paiva, E. L., Jr, J. M., \& Fensterseifer, J. E. (2009). Estratégia de produção e de operações. Porto Alegre: Bookman.

Scur, G., \& Queiroz, R. P. (Junho de 2017). O impacto da diversificação na estratégia de operações de empresas de bens de capital. Gestão \& Produção, 24. Acesso em 8 de Agosto de 2020, retirado de https://www.scielo.br/scielo. php?script $=$ sci_arttext\&pid $=$ S0104-530X2017000200206\&lng=pt\&tlng $=$ pt \#B016

Sumathi, S., \& Surekha, P. (2007). LabVIEW basedAdvanced Instrumentation Systems. New York: Springer.

Vilela, C. A., Medeiros, K. T., \& Piazza, M. J. (2014). Desenvolvimento de Software em LabVIEW para controle de um braço robótico e uma base móvel. São José dos Campos, São Paulo. 